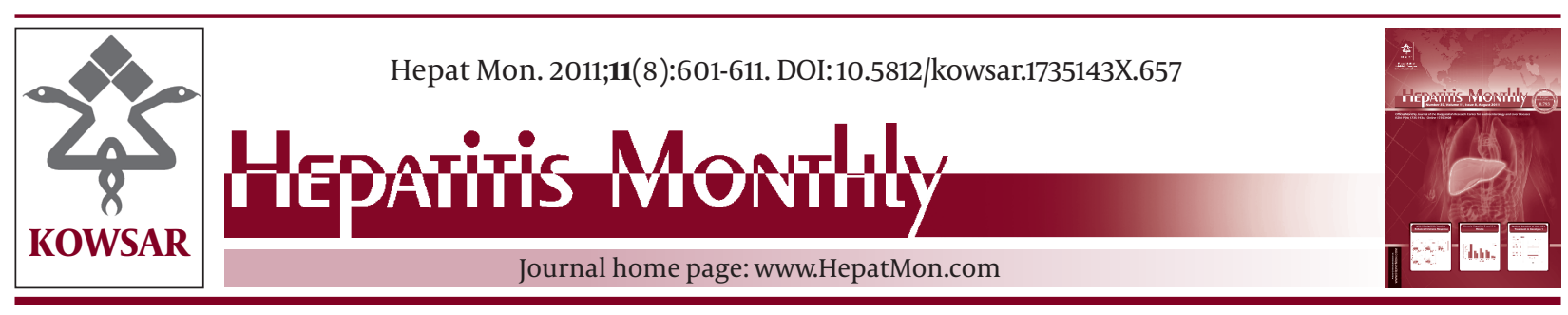

\title{
Recent Advances in the Management of Chronic Hepatitis B
}

\author{
Soo Ryang Kim ${ }^{1^{*}}$, Jisin Yang $^{1}$, Masatoshi Kudo ${ }^{2}$, Okio Hino $^{3}$ \\ ${ }^{1}$ Department of Gastroenterology, Kobe Asahi Hospital, Kobe, Japan \\ ${ }^{2}$ Department of Gastroenterology and Hepatology, Kinki University School of Medicine, Osakasayama, Japan \\ ${ }^{3}$ Department of Pathology and Oncology, Juntendo University, Tokyo, Japan
}

\begin{tabular}{l}
\hline A R T I C L E I N F O \\
\hline Article Type: \\
Review Article \\
\hline Article history: \\
Received: 24 Jan 2011 \\
Revised: 24 Feb 2011 \\
Accepted: 06 Mar 2011 \\
\hline
\end{tabular}

Keywords:

Hepatitis B virus

Interferon alpha

Nucleotide analogues

Entecavir

Tenofovir

\begin{abstract}
A B S T R A C T
There are seven approved treatments for adults with chronic hepatitis B virus infection in the United States and European countries: interferon- $\alpha$, pegylated interferon- $\alpha$, lamivudine, adefovir dipivoxil, entecavir, telbivudine, and tenofovir disoproxil fumarate. At present, two new analogues, entecavir and tenofovir are recommended as the first line therapy by the guidelines of European Association for the Study of the Liver and American Association Study for the Liver Diseases. On the other hand, regarding interferon therapy, use of pegylated interferon- $\alpha$ is recommended as the first line therapy instead of standard interferon- $\alpha$ by both guidelines. Therefore, the main scientific interests and unmet medical needs for treatment of chronic hepatitis B have been narrowed down to long-term efficacy and safety of the two said analogues-entecavir and tenofovir-and combination therapy of pegylated interferon- $\alpha$ with the two analogues. To put it concretely, further studies are needed to assess (1) the long-term efficacy and safety and resistance to entecavir and tenofovir; (2) the efficacy of different durations (24 weeks to 2 years) and lower doses of pegylated interferon- $\alpha$; (3) the role of combination therapy with two analogues to reduce resistance; and (4) the efficacy and safety of the two analogues with decompensated cirrhosis. Herein, we review the recent available data and results.

๑ 2011 Kowsar M.P.Co. All rights reserved.
\end{abstract}

- Implication for health policy/practice/research/medical education:

Several therapeutic modalities exist for the treatment of chronic hepatitis B. Based on recent available data, this review endeavors to present the long-term efficacy and safety of these modalities especially for entecavir and tenofovir as well as pegylated interferon- $\alpha$.

Please cite this paper as:

Kim SR, Yang J, Kudo M, Hino O. Recent Advances in the Management of Chronic Hepatitis B. Hepat Mon. 2011;11(8):601-11. [DOI: 10.5812] kowsar.1735143X.657]

\section{Introduction}

There are seven approved treatments for adults with chronic hepatitis B ( $\mathrm{CHB})$ in the United States and European countries: interferon (IFN) $\alpha$, pegylated (PEG) IFN- $\alpha$, lamivudine (LAM), adefovir dipivoxil (ADV), entecavir(ETV),telbivudine(TBV), and tenofovirdisoproxil fumarate (TDF). IFN- $\alpha$ and LAM have been approved for children with hepatitis B virus (HBV) infection. Two different treatment strategies are applicable in both

\footnotetext{
* Corresponding author at: Soo Ryang Kim, Department of Gastroenterology, Kobe Asahi Hospital, 3-5-25 Bououji-Cho, Nagata-Ku, 653-0801, Kobe, Japan. Tel:+81-786125151, Fax:+81-786125152.

E-mail: asahi-hp@arion.ocn.ne.jp

DOI: 10.5812/kowsar.1735143X.657

Copyright $\odot 2011$, BRCGL, Published by Kowsar M.P.Co. All rights reserved.
}

HBeAg-positive and negative CHB patients: treatment with PEG IFN- $\alpha$ and long-term treatment with NUCs. There are several treatment options for patients, making rational choices for the first and second line treatment sometimes difficult. Although available randomized controlled trials show encouraging short-term results demonstrating a favorable effect of these agents on intermediate markers of the disease such as HBV DNA level, liver enzyme tests, and liver histology, limited rigorous evidence exists demonstrating the effect of these therapies on important long-term clinical outcomes, such as the development of hepatocellular carcinoma or a reduction in mortality rate. Questions therefore remain about which groups of patients benefit from therapy and at which point in the course of disease 
this therapy should be initiated.

Herein, we pooled the available data focusing on longterm efficacy and safety of two new analogues-entecavir and tenofovir-and combination therapy of PEG INF- $\alpha$ and the said two analogues. In the first section, we summarize recent findings based on the consensus of the guidelines of the European Association for the Study of the Liver (EASL) and the American Association Study for the Liver Diseases (AASLD) $(1,2)$. In section two, presentations at EASL and AASLD annual meetings in 2010 are reported.

\section{Section I: Published Results Based on the Consensus of EASL and AASLD Guidelines \\ 2.1. Assessment of Outcomes}

Although various monitoring practices have been recommended, no clear evidence exists for an optimal approach. One proposed that the managementalgorithm used during therapy involves measuring HBV DNA and ALT levels every 12 weeks and HBeAg or anti-HBe levels every 24 weeks in patients who are HBeAg-positive. For patients who are HBeAg-positive and achieve a complete response (undetectable HBV DNA), seroconversion to anti-HBe may offer the opportunity to discontinue therapy after 6-12 months of "consolidation." During this period, regular monitoring of HBV DNA and HBeAg status should be done because relapse remains a possibility. HBsAg should be checked at 6-month intervals after $\mathrm{HBe}$ seroconversion if $\mathrm{HBV}$ DNA is undetectable. Quantitative HBsAg assay is still a research tool. HBeAg-negative patients should be similarly monitored for efficacy and safety through 48 weeks of treatment. A virological response with HBV DNA $<2000$ $\mathrm{IU} / \mathrm{mL}$ (approximately 10,000 copies/mL), i.e. 3.3 log10 IU/ $\mathrm{mL}$, is generally associated with remission of the liver disease. Undetectable HBV DNA in real-time PCR is the ideal desired of treatment sustained response with a high probability of HBsAg loss in the longer term. HBsAg should be checked at 6-month intervals if HBV DNA is undetectable. All patients treated with PEG IFN- $\alpha$ should be monitored for the known adverse effects of IFN. The balance of benefits and harms associated with screening for hepatocellular carcinoma is unknown and is an area for future research.

\subsection{Antiviral Effect of NUCs}

Table 1 summarizes the efficacy of NUCs treatment in a 48-week large randomized controlled trial with HBeAgpositive and -negative patients.

\subsubsection{Lamivudine (LAM)}

In large registration trials, both on HBeAg-positive and -negative patients with $\mathrm{CHB}$ and those with previous IFN failure, a daily dose of $100 \mathrm{mg}$ of LAM was compared to $0.5 \mathrm{mg}$ of ETV. LAM treatment for 48 weeks resulted in suppression of HBV DNA by an average of 5.4 log10 copies/ $\mathrm{mL}$ in HBeAg-positive patients and $4.5 \log 10$ copies/mL in HBeAg-negative patients. HBeAgseroconversion occurred in $18 \%$ of patients, rendered HBV DNA undetectable (<102 copies $/ \mathrm{mL}$ ) in 36\% (HBeAg-positive) to $72 \%$ (HBeAgnegative) of patients $(3,4)$.

\subsubsection{Adefovir (ADV)}

In 48-week registration trials, $\mathrm{CHB}$ patients who were HBeAg-positive and -negative received $10 \mathrm{mg} /$ day of ADV. ADV suppressed HBV DNA by $3.5 \log 10$ copies/mL in HBeAg-positive patients and 3.9 log10 copies/mLin HBeAgnegative; HBV DNA decreased to an undetectable level (< 102 copies $/ \mathrm{mL}$ ) in only $21 \%$ of HBeAg-positive patients and to $51 \%$ of HBeAg-negative patients; suppression of HBV DNA was relatively slow; it was also less likely to induce HBeAg seroconversion $(12 \%)(5,6)$.

\subsubsection{Entecavir (ETV)}

A daily dose of $0.5 \mathrm{mg}$ of ETV was found to be superior to $100 \mathrm{mg}$ of LAM in terms of suppression of HBV DNA by $6.9 \log 10$ copies/mL in HBeAg-positive patients and by $5.0 \log 10$ copies/mL in HBeAg-negative patients.

\begin{tabular}{|c|c|c|c|c|c|}
\hline $\begin{array}{l}\text { Treatment } \\
\text { Group }\end{array}$ & $\begin{array}{l}\text { HBeAg } \\
\text { Status }\end{array}$ & $\begin{array}{l}\text { HBV DNA } \\
\text { Suppression } \\
\text { (log10 copies/mL) }\end{array}$ & $\begin{array}{l}\text { HBV DNA } \\
\text { Undetectable, \% }\end{array}$ & ALT Normalization \% & HBeAg Serocon- Version \% \\
\hline \multirow[t]{2}{*}{$\operatorname{LAM}^{\mathrm{a}}(3,4)$} & Positive & -5.4 & 36 & 60 & 18 \\
\hline & Negative & -4.5 & 72 & 71 & - \\
\hline \multirow[t]{2}{*}{$\operatorname{ADV}^{\mathrm{a}}(5,6)$} & Positive & -3.5 & 21 & 48 & 12 \\
\hline & Negative & -3.9 & 51 & 72 & - \\
\hline \multirow[t]{2}{*}{$\operatorname{ETV}^{\mathrm{a}}(3,4)$} & Positive & -6.9 & 67 & 68 & 21 \\
\hline & Negative & -5.0 & 90 & 78 & - \\
\hline \multirow[t]{2}{*}{$\mathrm{TBV}^{\mathrm{a}}(7)$} & Positive & -6.4 & 60 & 77 & 23 \\
\hline & Negative & -5.2 & 88 & 74 & - \\
\hline \multirow[t]{2}{*}{$\mathrm{TDF}^{\mathrm{a}}(8)$} & Positive & $-4.5(12 w)$ & 76 & 68 & 21 \\
\hline & Negative & $-3.0(12 w)$ & 93 & 76 & - \\
\hline
\end{tabular}

a Abbreviations: ADV, Adefovir; ETV, Entecavir; LAM, Lamivudine; TDF, Tenofovir; TVB, Telbivudine 
Therapy with ETV was more likely to decrease HBV DNA to undetectable levels ( $<102$ log10 copies/mL) than LAM in $67 \%$ of HBeAg-positive patients and in $90 \%$ of $\mathrm{HBeAg}$ negative of patients. Histological improvement was achieved in $72 \%$ of ETV-treated patients compared to $62 \%$ in LAM-treated patients (HBeAg positive); and in 70\% of ETV-treated vs. $61 \%$ of LAM-treated patients (HBeAgnegative). The two drugs, however, did not differ in rates of HBeAg seroconversion-21\% vs. 18\%. Treatment effects were maintained with long-term ETV therapy, with HBeAg seroconversion rates increasing progressively to $31 \%$ at year two and 39\% at year three. In addition, at the end of year two, HBsAg loss was recorded in 5\% of ETV-treated and $2 \%$ of LAM-treated patients $(9,10)$. In the study of 96 weeks of treatment with $0.5 \mathrm{mg}$ of ETV in naïve Japanese patients, resistance was reported in only 1.7\% (11).

\subsubsection{Telbivudine (TBV)}

TBV is a potent L-nucleoside that is believed to cause chain termination and is highly potent against HBV in cell culture. TBV (600 mg/day) was superior to LAM (100 $\mathrm{mg}$ /day) in suppressing HBV DNA to undetectable levels of $<102$ copies $/ \mathrm{mL}$ (60\% vs. $40 \%$; a reduction from $6.4 \log 10$ to $5.5 \log 10$ copies $/ \mathrm{mL}$ ), and in achieving histological improvement (65\% vs. 56\%) but not in normalization of ALT (77\% vs. $75 \%$ ) or serological responses (HBeAg seroconversion in $23 \%$ vs. 22\%). In HBeAg-negative patients, TBV (600 mg/day) was superior to LAM (100 $\mathrm{mg}$ /day) in suppressing HBV DNA to undetectable levels (88\% vs. 71\%; reduction from 5.2 log10 to 4.4 log10 copies/ $\mathrm{mL}$ ) but not in achieving histological (67\% vs. 66\%) or normalization of ALT (74\% vs. 79\%) (7). These responses were well maintained during the second year of therapy, and HBeAg seroconversion increased to $30 \%$ by the end of year two (12).

\subsubsection{Tenofovir (TDF)}

In two 48-week randomized controlled trials, oral TDF (300 mg/day) was compared to ADV (10 mg/day) in treatment-naive patients with HBeAg-positive and -negative CHB. In HBeAg-positive patients, TDF reduced HBV DNA levels by $4.5 \log 10 \mathrm{IU} / \mathrm{mL}$ (12 weeks results) and suppressed HBV DNA to undetectable levels (<102 IU/mL) in $76 \%$ of patients vs. in only $13 \%$ in the ADV group. TDF and ADV treatments resulted in similar rates of histological benefit (74\% vs. 68\%) and HBeAg seroconversion (21\% vs. $18 \%$ ). An important finding was HBsAg loss in 3\% of patients during the first 48 weeks of therapy in the TDF group (8). In the HBeAg-positive group, at the end of year two of continuous TDF treatment, HBeAg seroconversion increased to $27 \%$ and HBsAg loss increased to $6 \%$ (13). In HBeAg-negative patients, TDF reduced HBV DNA levels by $3.0 \log 10 \mathrm{IU} / \mathrm{mL}$ (12 weeks results) and suppressed HBV DNA to undetectable levels $(<102 \mathrm{IU} / \mathrm{mL})$ in $93 \%$ of patients vs. in only $63 \%$ in the ADV group.

\subsection{Antiviral Resistance to NUCs}

Frequencies of antiviral resistance within five years of administration for the five NUCs are shown in Table 2. Although LAM has the most extensive safety record, its current use is limited by the high frequency of LAM resistance (24\% in year one, and $70 \%$ in year four) (14), and the availability of more potent agents with superior efficacy and markedly improved resistance profiles.

ADV is more expensive than TDF, is less effective, and produces higher rates of resistance. Although resistance to $\mathrm{ADV}$ is slow to emerge, resistant variants increase progressively after the first year, reaching $29 \%$ in year five (16). The advantages of ADV are its limited resistance during first two years, the absence of cross-resistance with LAM and other L-nucleosides and, therefore, its value as treatment for LAM-resistant CHB $(17,18)$ and for hepatic decompensation associated with LAM resistance prior to and after liver transplantation (19). The high potency and excellent safety profile of ETV are complemented by its very high barrier to resistance in treatment-naive patients $(<1 \%)$ in year four. ETV and TDF are potent HBV inhibitors and they have a high barrier to resistance (3, $20,21)$. Therefore, they can be confidently used as the

\begin{tabular}{lll}
\hline Table 2. Frequency of Antiviral Resistance to Nucleoside Analogs Treatment & \\
\hline Treatment Group & Treatment Duration, $\mathbf{y}$ & Antiviral-resistance, \% \\
\hline $\operatorname{LAM}^{\mathrm{a}}(14)$ & 1 & 24 \\
& 2 & 42 \\
& 3 & 53 \\
$\operatorname{ADV}^{\mathrm{a}}$ (naive patients) (6) & 4 & 70 \\
& 1 & 0 \\
& 2 & 3 \\
& 3 & 11 \\
$\operatorname{ETV}^{\mathrm{a}}$ (naive patients) (10) & 4 & 18 \\
$\operatorname{TBV}^{\mathrm{a}}$ (naive patients) (7, 12) & 5 & 29 \\
& 4 & $<1$ \\
$\operatorname{TDF}^{\mathrm{a}}$ (15) & 1 & $2-5$ \\
\hline
\end{tabular}

a Abbreviations: LAM, Lamivudine; ADV, Adefovir; ETV, Entecavir; TVB, Telbivudine; TDF, Tenofovir 
first-line monotherapies. The role of monotherapy with ETV or TDF could be modified if higher rates of resistance become apparent with longer treatment duration. In a study of 96 weeks of treatment with TDF, no evidence of TDF resistance was found (15).

TBV is a potent inhibitor of HBVbut, due to a low genetic barrier to resistance, a high incidence of resistance has been observed in patients with high baseline levels of replication and in those with detectable HBV DNA after 24 weeks of therapy. In a large registration trial, TBV was compared to LAM in HBeAg-positive and -negative patients. The frequency of antiviral resistance to TBV at one year was $5 \%$ of HBeAg-positive and in only $2 \%$ of HBeAg-negative patients (7).

Virological breakthrough in compliant patients is related to viral resistance. Resistance is associated with prior treatment with NUCs (i.e., LAM, ADV, TBV, emtricitabine) or, in treatment-naive patients, with high baseline HBV DNA levels, a slow decline in HBV DNA and partial virological response during treatment. Resistance should be identified as early as possible before clinical breakthrough (i.e. increased ALT) by means of HBV DNA monitoring; if possible, the pattern of resistance mutations should be identified to adapt therapeutic strategies. Indeed, clinical and virological studies have demonstrated the benefit of an early treatment adaptation-as soon as viral load increases $(22,23)$. In case of resistance, an appropriate rescue therapy should be initiated with the most effective antiviral effect and the minimal risk to induce multiple drug-resistant strains. Therefore, adding-on a second drug without cross-resistance is the only efficient strategy.

- LAM resistance: Add TDF (add ADV if TDF is not yet available).

- ADV resistance: It is recommended to switch to TDF if available and add a second drug without crossresistance. If an N236T substitution is present, add LAM, ETV or TBV or switch to TDF plus emtricitabine. If an A181T/V substitution is present, add ETV(the safety of the TDF-ETV combination is unknown) or switch to TDF plus emtricitabine.

- TBV resistance: Add TDF (add ADV if TDF is not yet available). The long-term safety of these combinations is unknown.

- ETV resistance:Add TDF(the safety of this combination is unknown).

- TDF resistance: Resistance to TDF has not been described so far. It is recommended that genotyping and phenotyping be done by an expert laboratory to determine the cross-resistance profile. ETV, TBV, LAM or emtricitabine could be added (the safety of these combinations is unknown).

\subsection{Long-term Therapy with NUCs}

HBV DNA levels should be monitored at week 12 to ascertain virological response and then every 12 to 24 weeks. HBV DNA reduction to undetectable levels by real- time PCR (i.e. $<10-15 \mathrm{IU} / \mathrm{mL}$ ) should ideally be achieved to avoid resistance. HBV DNA monitoring is thus crucial to detect treatment failure. In HBeAg-positive patients, HBeAg and subsequently anti-HBe antibodies once HBeAg is negative should be measured at intervals of 6 to 12 months. NUCs are cleared by the kidneys, and appropriate dose adjustments are recommended for patients with reduced creatinine clearance. Drug concentrations are comparable in patients with varying degrees of hepatic impairment but this has not been fully studied. Exacerbations of hepatitis B may occur and require more intensive monitoring (monthly in the first three months) in patients with cirrhosis. The onset of complications in these patients requires urgent management. Renal impairment has rarely been reported in patients with HIV infection receiving anti-HBV drugs, or in patients receiving nephrotoxic drugs and treated with TDF or ADV, thus, appropriate monitoring for nephrotoxicity and dose adjustments are necessary.

Long-term monitoring for carcinogenesis with ETV is ongoing. Myopathy has rarely been reported in $\mathrm{CHB}$ patients treated with TBV. Peripheral neuropathy has been observed in patients treated with PEG IFN and TBV and thus this combination should be avoided.

\subsection{Treatment with PEG IFN- $\alpha$}

The main theoretical advantages of IFN- $\alpha$ (conventional or PEG) are the absence of resistance and the potential for immune-mediated containment of HBV infection with an opportunity to obtain a sustained virological response off-treatment and a chance of HBsAg loss in patients who achieve and maintain undetectable HBV DNA. Frequent side effects and subcutaneous injection are the main disadvantages of IFN- $\alpha$ treatment. IFN- $\alpha$ is contraindicated in patients with decompensated HBVrelated cirrhosis or autoimmune disease and in those with uncontrolled severe depression or psychosis. Full information about the advantages, adverse events and inconveniences of PEG IFN- $\alpha$ vs. NUCs should be provided so the patient can participate in the decision. There has been a resurgence of interest in IFN therapy the past five years, largely based on results of large clinical trials demonstrating that PEG IFN has more potent antiviral activity than standard IFN- $\alpha$ and that in contrast to NUCs, it does not result in any antiviral resistance and can be given for a finite period rather than indefinitely. Therefore, when compared to the standard IFN $\alpha$-2a in a dose of 4.5 million units three times weekly, PEG IFN in a dose of $180 \mu \mathrm{g}$ once weekly for 12 months resulted in a greater decline in HBV DNA levels and a higher rate of HBeAg seroconversion (33\% vs. 25\%) (24). Three large multicenter trials of PEG IFN therapy have been published-two in HBeAg-positive $(25,26)$ and one in HBeAg-negative CHB patients (27). Each study included treatment arms in which PEG IFN was used alone or in combination with LAM. Two studies used PEG IFN $\alpha$-2a and one used PEG IFN $\alpha-2 b$. The results 24 weeks after 
treatment are shown in Table 3.

In a multinational European study, PEG IFN $\alpha-2 b$ was given in a dose of $100 \mu \mathrm{g}$ weekly for 32 weeks followed by $50 \mu \mathrm{g}$ weekly until completion of 52 weeks of treatment with or without LAM (100 mg daily) in 266 patients who were HBeAg-positive (25). Seroconversion of HBeAg by six months after treatment occurred in similar proportions of patients receiving monotherapy as combination therapy ( $29 \%$ vs. $29 \%)$ as did loss of HBsAg (7\% vs. 7\%). Suppression of HBV DNA levels and normal ALT values or HBV DNA levels below 20,000 copies/mL was significantly higher with PEG IFN monotherapy (59\% and 43\%, respectively) than with 48 weeks of LAM monotherapy ( $44 \%$ and $29 \%$, respectively). Again, the addition of LAM to PEG IFN did not appear to increase the response rates even though there was greater HBV DNA suppression in combination therapy. Furthermore, 3\% (12/356) of patients who received PEG IFN but none of 181 patients who received LAM alone became HBsAg-negative.

\begin{tabular}{|c|c|c|c|c|c|c|}
\hline HBeAg Status & Treatment Arms & No. & $\begin{array}{l}\text { HBV DNA } \\
\text { Suppression, \% }\end{array}$ & $\begin{array}{l}\text { HBV DNA } \\
\text { Undetectable, } \\
\%\end{array}$ & $\begin{array}{l}\text { ALT } \\
\text { Normalization, } \\
\%\end{array}$ & $\begin{array}{l}\text { HBeAg } \\
\text { Seroconversion, } \\
\%\end{array}$ \\
\hline \multirow[t]{2}{*}{ Positive (25) } & $\begin{array}{l}\text { PEG IFN } 100 \mu \mathrm{g} / \mathrm{wk} \times 32 \\
\mathrm{wk} \text { ? } 50 \mu \mathrm{g} \times 20 \mathrm{wk}\end{array}$ & 136 & 27 & 7 & 32 & 29 \\
\hline & 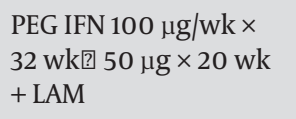 & 130 & 32 & 9 & 35 & 29 \\
\hline \multirow[t]{3}{*}{ Positive (26) } & $\begin{array}{l}\text { PEG IFN } 180 \mu \mathrm{g} / \mathrm{wk} \times \\
48 \mathrm{wk}\end{array}$ & 271 & 32 & 14 & 41 & 32 \\
\hline & $\begin{array}{l}\text { PEG IFN } 180 \mu \mathrm{g} / \mathrm{wk}+ \\
\text { LAM } 48 \mathrm{wk}\end{array}$ & 271 & 34 & 14 & 39 & 27 \\
\hline & LAM for $48 \mathrm{wk}$ & 272 & 22 & 5 & 28 & 19 \\
\hline \multirow[t]{3}{*}{ Negative (27) } & $\begin{array}{l}\text { PEG IFN } 180 \mu \mathrm{g} / \mathrm{wk} \times \\
48 \mathrm{wk}\end{array}$ & 177 & 43 & 19 & 59 & - \\
\hline & $\begin{array}{l}\text { PEG IFN } 180 \mu \mathrm{g} / \mathrm{wk}+ \\
\text { LAM } 48 \mathrm{wk}\end{array}$ & 179 & 44 & 20 & 60 & - \\
\hline & LAM for $48 \mathrm{wk}$ & 181 & 29 & 7 & 44 & - \\
\hline
\end{tabular}

loss of HBeAg were greater on combination therapy than monotherapy, but relapse rates were higher in the group that received LAM so that sustained responses six months after stopping treatment were equivalent. A comparison group receiving LAM alone was not included. In a second larger multicenter trial, a total of 814 patients with $\mathrm{HBeAg}$-positive $\mathrm{CHB}$ were given either PEG IFN $\alpha$-2a alone (180 $\mu$ g once weekly), LAM alone (100 mg daily), or the combination for 48 weeks (26). Again, HBV DNA suppression was greater in patients receiving combination therapy than in those receiving either PEG IFN or LAM monotherapy. However, rates of HBeAg seroconversion six months after discontinuation of therapy was greater with PEG IFN than LAM monotherapy (32\% vs. 19\%) and was no higher with combination therapy (27\%). Loss of HBsAg occurred in 16 of 542 (3\%) patients who received PEG IFN (alone or with LAM) but in none of 272 patients receiving LAM alone $(P=0.004)$.

Finally, in another large multicenter trial, patients with HBeAg-negative hepatitis B were treated with PEG IFN $\alpha$-2a alone (180 $\mu$ g once weekly), LAM alone (100 mg daily), or the combination for 48 weeks (27). Six months after stopping therapy, the percentage of patients with
These three studies showed that a one-year course of PEG IFN induced HBeAg seroconversion in about onethird of HBeAg-positive patients and induced a lasting biochemical and virological response in almost $40 \%$ of HBeAg-negative patients. Furthermore, therapy with PEG IFN led to loss of HBsAg in a small proportion of patients, an outcome not seen with a one-year course of LAM therapy. Adding LAM to PEG IFN did not increase the rate of sustained responses. These results suggested that a trial of one-year course of PEG IFN might be appropriate in selected patients with $\mathrm{CHB}$, before embarking on longterm suppressive therapy with NUCs.

\section{Section II. Topics of EASL and AASLD Presentations at Annual Meetings in 2010}

\subsection{Efficacy of NUCs}

\subsubsection{EASL Abstract 1009}

Effectiveness of ETV for NUC-naive HBeAg-negative CHB patients in clinical practice: A two-year multicenter cohort study on 311 patients. Lampertico P. 


\subsubsection{Key Results}

311 consecutive NUC-naive HBeAg-negative CHB patients, recruited in 17 Italian liver units, were treated with ETV $0.5 \mathrm{mg}$ for 23 months $(10,28)$.

- 294 (94\%) patients achieved a virological response (97\% at week 48).

- Two patients had primary non-response at week 12, and three (1\%) patients had a virological breakthrough. No ETV resistance in two patients and suboptimal compliance in one patient.

- Two (0.6\%) patients cleared HBsAg, seroconverted to anti-HBs and stopped ETV.

- No ETV-related serious adverse events were reported.

- 19 (6\%) patients had a partial virological response at week 48; 50\% with HBV DNA > $1000 \mathrm{IU} / \mathrm{mL}$. TDF + ETV inhibited HBV replication in the high viral load partial responders.

\subsubsection{Comments}

This cohort supports ongoing evidence that ETV was effective in this real life population of primarily HBeAgnegative patients, of whom almost $50 \%$ were with LC.

\subsubsection{AASLD PO 391}

Maintained long-term suppression of HBV replication in NUC-naive patients with $\mathrm{CHB}$ treated with ETV monotherapy in field practice: The Italian multicenter experience. Lampertico $\mathrm{P}$, et al.

- Virological responses increased over time in both HBeAg-positive and -negative patients, with more than 90\% achieving undetectable HBV DNA.

- HBV remained suppressed over time in the vast majority of patients; only $4 \%$ of patients showing a short lasting virological blip.

- Serological responses, i.e. HBeAg seroconversion and HBsAg loss, increased over time. Five patients stopped ETV successfully. And most patients developed a normal ALT level.

- Renal safety: Serum creatinine increased in few patients ( $<1 \%)$-an event not considered drug-related.

- Patient retention rates were $84 \%$.

\subsubsection{Comments}

This reconfirms ETV monotherapy suppresses HBV replication in most NUC-naive patients in real practice up to 30 months, independently of serology and safety profile was consistent with registration studies.

\subsubsection{AASLD PO 369}

Effectiveness and safety of TDF in field practice: A multicenter European cohort study of 737 patients with CHB. Lampertico P, et al.

- Most NUC-naive patients achieved undetectable HBV DNA by PCR assay and developed a normal ALT level. Viral suppression was however significantly faster in patients with lower baseline viremia.

- Primary non-response at week 12 and partial virological response at week 48 occurred in 3\% and $18 \%$ of the patients, respectively.

- Eight patients seroconverted to anti-HBe with an 18month cumulative probability of $32 \%$ and two cleared HBsAg.

- In NUC-experienced patients, HBV DNA became undetectable in almost $74 \%$ of the patients, independently of treatment regimen (TDF vs. TDF + LAM).

- No major changes of renal function (glomerular and tubular) were observed over 18 months of treatment. Dose adjustments, hypophosphatemia and increased phosphate wasting occurred more frequently in NUCexperienced patients.

\subsubsection{Conclusions}

TDF suppressed HBV replication in most NUC-naive and NUC-experienced patients in field practice up to 18 months. The safety profile was favorable with few patients, mainly NUC-experienced, showing some degrees of renal dysfunction.

\subsubsection{Comments}

This adds another clinical data of TDF. Data on renal toxicity seem not to be consistent. Therefore, we will wait and see the accumulation of more data.

\subsection{Resistance Data}

\subsubsection{AASLD PO 1365}

No resistance to TDF was detected in following up to 192 weeks of treatment in patients mono-infected with $\mathrm{CHB}$ virus. Snow-Lampart A, et al. This evaluated NUC-naive, 176 HBeAg+ and $250 \mathrm{HBeAg-}$ patients on TDF treatment up to four years. No resistance was detected in 348 patients at 192 weeks. Some doctors chose to add emtricitabine (FTC) $200 \mathrm{mg}$ to TDF for seven patients from the 3rd year and five from the 4 th year on, if patients were confirmed to be viremic at week 72 or beyond. They reported good tolerability of TDF.

\subsubsection{Comments}

TDF is shown to be free from resistance in this clinical trial setting. It needs to be examined in real practice usage. (cf. ETV has been presented to have $1.2 \%$ resistance rate in five years as shown in 2009 EASL guidelines).

\subsection{Safety Evaluation of NUCs}

\subsubsection{Long-term Data}

\subsubsection{EASL Poster 1016}

Low rates of nucleos(t)ide-associated adverse events in the long-term experience with ETV. Manns M, et al. 


\subsection{Key Results}

Long-term safety data from the roll-over study ETV901 are reviewed, focusing on adverse events (AEs) with a potential nucleos(t)ide association. Median exposure to ETV in ETV-901 was 184 weeks (almost 3.5 years) (Table 4). Of the 1,051 patients in this analysis, 448 (46\%) had prior ETV exposure in previous studies. Overall, the most common AEs (related and unrelated) were upper respiratory tract infection (27\%), headache $(20 \%)$ and nasopharyngitis (16\%). Lactate increase or bicarbonate decrease occurred in six $(<1 \%)$ patients and no cases of lactic acidosis syndrome were reported. Rates of serious AEs, discontinuations due to AEs, liver disease progression and ALT flares were consistent with previous Phase III observations. AEs typically associated with NUCs use were reported infrequently by investigators. Study ETV-901 demonstrates that ETV is generally a welltolerated treatment at a dose of $1.0 \mathrm{mg} /$ day when used to treat a diverse population of $\mathrm{CHB}$ patients.

\subsection{Comments}

This roll-over study ETV-901 provides an opportunity to assess safety events in a large cohort of diverse CHB patients, 1051 patients who received long-term ETV (1.0 $\mathrm{mg} /$ day) therapy in the study over a median of 184 weeks. Study ETV-901 demonstrates that ETV is a generally welltolerated long-term treatment.

\subsubsection{Renal Data}

\subsubsection{EASL Abstract 1007}

Risk of renal toxicity with TDF for CHB. Gish R, et al.

\subsection{Key Results}

84 patients on TDF (either monotherapy or in combination with another antiviral drug) were matched by age ( \pm 5 years) and gender to 84 ETV monotherapy patients.

- TDF was shown to be well tolerated: Serum creatinine increases of $0.2 \mathrm{mg} / \mathrm{dL}$ were found to be common,

\begin{tabular}{ll}
\hline $\begin{array}{l}\text { Table 4. Adverse Event (AE) Results from } 901 \text { Studies (Mean of 184 Weeks of } \\
\text { Treatment)( } \mathrm{n}=1051 \text { ) }\end{array}$ \\
\hline Adeverse Events (AEs) & Total, No.(\%) \\
\hline Any AEs & $900(86)$ \\
Serious AEs & $169(16)$ \\
Discontinuations due to AEs & $14(1)$ \\
Grade 3-4 AEs & $203(19)$ \\
Grade 3-4 AEs considered related to ETV & $45(4)$ \\
All deaths & $27(3)$ \\
Liver-related deaths & $12(1)$ \\
Non-liver-related deaths & $15(1)$ \\
\hline
\end{tabular}

whereas such an increase was rare for the TDF arm and less than ETV group $(2 \%, P=0.029)$ probably due to a significantly higher rate of dose adjustments.

- History of diabetes, and transplant, significantly increased the risk of renal injury in all CHB patients $(P=$ 0.004 , and 0.002, respectively).

\subsection{Comments}

This is a presentation with the risk of TDF with nephrotoxicity. The study, however suffers from some limitations including, retrospective analysis of data that may cause a selection bias for patients with renal problems to begiven ETVas TDFwas given as monotherapy or in combination therapy. Patients on ETV had longer duration of disease and comorbidities were not equally matched between the study arms. Furthermore, dose of TDF were often adjusted.

\subsubsection{EASL Abstract 1010}

OPTIB study: A multicenter prospective open label study on TDF for CHB patients with suboptimal response to ADV or ADV+LAM treatment. Levrero M, et al.

\subsection{Key Results}

Adults with HBV monoinfection and HBV DNA > 103 copies/mL after 48 weeks of ADV with or without LAM were enrolled and switched to TDF $300 \mathrm{mg}$ daily with or without LAM.

- 91 patients were screened and 85 were enrolled. 13 (15\%) patients were switched from ADV to TDF and 72 (85\%) to TDF + LAM combination.

- The median duration of prior ADV therapy was 29.2 months.

- At 24 weeks of treatment, median HBV DNA fall from baseline was $2.02 \log 10 \mathrm{IU} / \mathrm{mL}$ and $62 \%$ had HBV DNA levels $<69 \mathrm{IU} / \mathrm{mL}$ and $49 \%$ HBV DNA levels $<12 \mathrm{IU} / \mathrm{mL}$.

- At 48 weeks, $81 \%$ of patients had HBV DNA levels $<69$ $\mathrm{IU} / \mathrm{mL}$ and $65 \%$ had HBV DNA levels $<12 \mathrm{IU} / \mathrm{mL}$.

- The proportion of patients reaching negativity through 48 weeks was not correlated with HBeAg status or the presence of ADV resistance mutations at the baseline.

- No clinically significant side effects related to TDF were reported.

\subsection{Comments}

Despite the concerned nucleotide cross-resistance profile, this study showed higher response rates than other presented data sets. This study implies that TDF can be used to salvage patients exposed to ADV and/or ADV + LAM.

\subsubsection{EASL Abstract 1028}

Renal safety and antiviral efficacy of TDF monotherapy in nucleos(t)ide analogue refractory patients with 
hepatitis B virus (HBV) mono-infection. vanBommel F, et al.

\subsection{Key Results}

Data from all HBV monoinfected patients treated with TDF monotherapy in 19 European centers were retrospectively analyzed. Of 343 patients screened, 195 were found eligible for retrospective data analyses; 137 were HBeAg-positive. The mean \pm SD HBV DNA level was $6.9 \pm 1.5$ (range 4-10) $\log 10$ copies/mL.

- After 48 months of TDF therapy a mean decrease of estimated glomerular filtration rate (eGFR) of $9 \%$ was observed.

- During the total observation period, 10 patients had a moderate decrease (20\%-30\%) in eGFR; six patients had a severe decrease (>30\%), however eGFR remained within normal values in most patients and did not decrease to $<$ $50 \mathrm{~mL} / \mathrm{min}$ in patients with initially normal eGFR values.

- TDF dosage did not need to be adjusted due to changes in creatinine.

- A model assessing the influence of age on the eGFR rates as determined by the MDRD formula confirmed a mild decrease in eGFR driven by increase in serum creatinine during the 48 months.

- A comparison of the mean eGFR rates in the TDF group and the control group showed no significant differences in eGFR decrease.

\subsection{Comments}

In this ongoing real world, independent cohort study evaluating TDF in refractory patients, it was shown that TDF is not associated with renal issues, though this study has excluded patients with higher risk of renal toxicity including, concomitant comorbidities-i.e. those with kidney disease, arterial hypertension, and/or diabetes.

\subsubsection{AASLD PO 393}

Prevalence of renal alterations indicative of proximal tubular damage(PTD)in patients with $\mathrm{CHB}$ virus infection during long-term therapy with TDF. vanBommel, et al.

\subsection{Summary}

In total, 24 of 61 (39\%) patients showed at least one sign of PTD, which would be either renal phosphate loss (hypophosphatemia and/or TmPO4/GFR $\downarrow$ ), glucosuria or increased $\alpha 1$-microglobulin/creatinine ratio.

\subsection{Conclusion}

This study confirms that long-term treatment with TDF does not lead to a significant decrease in eGFR in HBVinfected patients, regardless of age or risk factors for kidney dysfunction. However, signs of PTD were prevalent in $39 \%$ of patients after mean treatment duration of
29 months. As there were no samples from baseline available, there was no clear association between these alterations and the use of antiviral agents. Therefore, further follow-up data are needed to determine the role of TDF therapy in possible proximal tubular damage. More specific markers may help to further determine the drug's influence on renal function.

\subsubsection{Bone Study \\ 3.3.3.1. AASLD PO 414}

High prevalence of reduced bone mineral density in patients with $\mathrm{CHB}$ under nucleos(t)ide analogues treatment. Vigano $\mathrm{M}$, et al. Single center (Universita di Milano), cross-sectional study studied 319 patients with CHB receiving NUC over a one-year period. Dual X-ray absorptiometry (DEXA) of the lumbar spine (LS) and femoral neck (FN) revealed that two thirds of CHB patients undergoing NUC treatment had reduced bone mineral density (BMD), osteoporosis at either LS or FN was present in 19\% and osteopenia in $49 \%$ of the patients. Multivariate analysis showed that female sex, older age and nucleotide (ADV and TDF) treatment were independently associated with a reduced BMD.

\subsection{Comments}

It is noteworthy that only nucleotides (ADV and TDF), not nucleosides (ETV and LAM), was associated with reduced BMD. Clinicians may need periodical screening of patients for osteoporosis.

\subsubsection{PEG IFN for $\mathrm{CHB}$ \\ 3.3.4.1. EASL Abstract 98}

Extended (two years) treatment with PEG INF $\alpha$-2a [40 $\mathrm{kD}$ ] improves sustained response rates in genotype $\mathrm{D}$ patients with HBeAg-negative CHB. Lampertico P, et al.

\subsection{Results}

PEG IFN $\alpha$-2a $180 \mu \mathrm{g} /$ week was evaluated for HBeAgnegative patients with $\mathrm{CHB}(\mathrm{n}=128)$ for its duration (48vs. 96 weeks) (Table 5). Virologic response was superior with 96 weeks and notably HBsAg loss retention was observed in $10 \%$ of patients one year after the therapy of 96 weeks. These edges NUCs in efficacy in this study population, but it should be noted that different genotypes would respond differently and we need further studies in patients with various backgrounds.

\subsection{Comments}

It is still in the experimental stage but this deserves to be examined further, although long-term treatment poses cost and safety concerns and may limit the number of eligible patients for this therapy. 


\begin{tabular}{lll}
\hline Table 5. Safety Profiles of Extended PEG IFN Therapy & & \\
\hline Safety Outcome & 48-Week PEG IFN ${ }^{\text {a }}$ (n= 51) & 96-Week PEG IFN (n= 52) \\
\hline$\geq 1 \mathrm{AE}^{\text {a }}$, & 82 & 77 \\
$\geq 1$ serious AE, \% & 14 & 6 \\
Need for dose reduction, \% & 31 & \\
Study withdrawal, \% & & 12 \\
$\quad$ Due to AEs & 16 & 12 \\
Reasons other than safety & 8 & 0 \\
Death ${ }^{\text {b }}$, No. & 1 & 0 \\
\hline
\end{tabular}

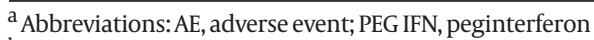

${ }^{\mathrm{b}}$ Patient died of hepatocellular carcinoma during follow-up

\subsection{Efficacy and Safety of NUCs in Decompensated Cirrhosis}

Decompensated cirrhosis is a serious complication of CHB. The five-year survival of patients with decompensated cirrhosis (14\%) has been reported and is lower than that for patients with compensated cirrhosis (84\%) (2). However, suppression of viral replication with antiviral therapy has been shown to result in clinical improvement and increased survival (2). There are limited data on safety and efficacy of NUC therapy in patients with $\mathrm{CHB}$ and decompensated cirrhosis. Summarized here are recently presented data including two randomized clinical trials $(28,29)$, and a cohort study on Korean patients pertaining to the use of ETV. in this patient population.

\subsubsection{EASL Oral Abstract 7}

Treatment of decompensated HBV-cirrhosis: results from a two-year randomized trial with telbivudine or lamivudine. Gane EJ, et al. (Table 6). This study was to evaluate clinical and virological outcomes of TBV $v s$. LAM in 232 patients (mean CTP and MELD score TBV 8.1 and 14.7; LAM 8.5 and 15.5). At baseline, the median age was almost 50 years-65\% Asian, almost $73 \%$ males, and approximately 57\% HBeAg-negative. This RCT showed that both therapies were safe but with high rates of rebounds/virological breakthroughs. There was only a limited improvement in MELD score of 0.2 with TBV, and 1 with LAM.

\subsubsection{Comments}

In this large scale study with long-term follow-up, TBV was well tolerated, stabilized liver function and had comparable tolerability to LAM. Safety profiles were similar between treatment arms, however, both TBV and LAM showing almost 30\% viral breakthrough. This result seems to reinforce the need to use potent antiviral treatment with low rates of resistance in this population with advanced disease, like ETV or TDF.

\subsubsection{EASL Abstract 1011}

Risk and predictors of mortality or hepatocellular carcinoma among ETV- or ADV-treated CHB patients with evidence of hepatic decompensation. Liaw Y, et al.

\subsubsection{Key Results}

This industry-sponsored study examined predictors of death and HCC in pooled data from ETV-treated and ADV-treated patients. The baseline predictors for death and HCC were examined in the 191 patients randomized to receiving either $1.0 \mathrm{mg} /$ day ETV or $10 \mathrm{mg} /$ day ADV for up to 96 weeks using univariate and multivariate Cox proportional hazard models with pooled data.Significant predictors of mortality in univariate analysis included serum creatinine level, MELD score, total bilirubin and albumin level. The multivariate analyses showed that a decreased hepatic function (increased bilirubin and decreased albumin level) is a significant predictor of mortality among CHB patients with decompensated liver disease treated with nucleos(t)ide analogues. Cumulative HCC rates were $12 \%$ and $20 \%$ among ETV-treated and ADVtreated patients, respectively. Cumulative death rates were $23 \%$ and 33\% among ETV-treated and ADV-treated patients, respectively. HBV genotype $\mathrm{B} / \mathrm{C}$ was the only predictor for development of HCC (Table 7).

\subsubsection{Comments}

The ETV-048 subanalysis reinforces the importance of

\begin{tabular}{|c|c|c|c|}
\hline 2-Year Outcome (ITT Population) & $\operatorname{TBV}^{a}(n=114)$ & LAM $^{a}(n=114)$ & Pvalue \\
\hline HBV DNA, \% (<300 copies/mL) & 49 & 40 & 0.15 \\
\hline Viral breakthrough, \% (HBV DNA > 1 log10 copies/mL above nadir) & 28 & 37 & 0.16 \\
\hline Composite endpoint, \% & 34 & 24 & 0.004 \\
\hline CTP score improved or stabilized, \% & 75 & 74 & NS ${ }^{a}$ \\
\hline
\end{tabular}

a Abbreviations: LAM, lamivudine; NS, not significant; TBV, telbivudin 


\begin{tabular}{|c|c|c|}
\hline & \multicolumn{2}{|c|}{ Week 48} \\
\hline & ETV $^{\mathbf{a}}$ & $\mathbf{A D V}^{\mathbf{a}}$ \\
\hline \multicolumn{3}{|l|}{ Cumulative efficacy of NUCs } \\
\hline $\begin{array}{l}\text { HBV DNA change from baseline, }(\text { log10 copies/mL) } \\
\text { HBV DNA, No. }(\%)(<300 \text { copies/mL) } \\
\text { HBeAg loss, No.\% } \\
\text { HBeAg seroconversion, No.\% } \\
\text { HBsAg loss, No.\% } \\
\text { CTP score improvement or no worsening, No.\% } \\
\text { CTP score, No.\% ( } \geq 2 \text {-point reduction) } \\
\text { MELD score change from baseline, Mean (SE) }{ }^{\text {a }}\end{array}$ & $\begin{array}{l}4.66 \\
57(100) \\
11(54) \\
6(54) \\
5(100) \\
61(100) \\
35(100) \\
2.6(0.62)\end{array}$ & $\begin{array}{l}3.90 \\
20(91) \\
18(51) \\
10(51) \\
0(91) \\
67(91) \\
27(91) \\
1.7(0.50)\end{array}$ \\
\hline \multicolumn{3}{|l|}{ Cumulative safety of NUCs } \\
\hline $\begin{array}{l}\text { Any AE, No.\% } \\
\text { Grade 3-4 AEs, No.\% } \\
\text { Deaths, No.\% } \\
\text { Serum Cr, No.\%, ( } \geq 0.5 \mathrm{mg} / \mathrm{dL} \text { increase) } \\
\text { HCC, No.\% } \\
\text { Discontinuation due to AEs, No.\% }\end{array}$ & $\begin{array}{l}91(89) \\
55(54) \\
23(23) \\
17(17) \\
12(12) \\
7(7)\end{array}$ & $\begin{array}{l}86(97) \\
42(47) \\
29(33) \\
21(24) \\
18(20) \\
5(6)\end{array}$ \\
\hline
\end{tabular}

${ }^{a}$ Abbreviations: ADV, adefovir dipivoxil; ETV, entecavir; SE, standard error

biologic risk factors (including baseline characteristics and inclusion criteria) as predictors associated with increased HCC and/or mortality in decompensated cirrhotic patients.

\subsection{Cohort Study in Korean Patients}

Shim JH et al. Efficacy of ETV in treatment-naive patients with HBV-related decompensated cirrhosis (30). This cohort study evaluated the effect of ETV monotherapy (0.5 mg QD for $\geq 12$ months) on viral suppression and hepatic function in 70 consecutive treatment-naive patients with $\mathrm{HBV}$-associated decompensated cirrhosis (defined as CTP $\geq 7$ [class B and C]), or the presence of portal hypertension complications). Comparator group consists of compensated LC patients with $\operatorname{HBV}(\mathrm{n}=144)$. Virologic response in this decompensated group $(n=55)$ was also compared to compensated cirrhosis. 15 patients in the decompensated group received ETV $<12$ months and therefore were not included in the comparative analysis with the compensated group. The baseline characteristics for decompensated and compensated groups were similar for gender ratio, HBV DNA levels (mean \pm SD for total patients was $7.34 \pm 1.43 \log 10$ copies/ $\mathrm{mL} ; \mathrm{n}=199$ ), and proportion HBeAg-positive (mean for total patients: $58.8 \% ; \mathrm{n}=199$ ).

However, in comparison to the compensated group, those with hepatic decompensation had a greater mean age (52.6 vs. 46.8 year, $P<0.001)$, lower mean \pm SD serum ALT (101.9 $\pm 110.7 v s .156 .5 \pm 160.5 \mathrm{IU} / \mathrm{L}, P=0.021)$; and higher mean \pm SD CTP $(8.1 \pm 1.7$ vs. $5.3 \pm 0.05)$ and MELD $(11.5 \pm 3.9$ vs. $7.0 \pm 1.5)$ scores $(P<0.001$ for both $)$.

Virologic, serologic and biochemical responses after 12 months of ETV therapy in the decompensated and compensated groups are presented in Table 8. Overall, at 12 months the rates for undetectable HBV DNA, HBeAg loss/seroconversion, and ALT normalization were not significantly different between the compensated and decompensated groups. In an intention-to-treat analysis of efficacy of all 70 patients with decompensated cirrhosis, the cumulative rates of HBV DNA undetectability and HBeAgloss at 12 months were $92.3 \%$ and $54.0 \%$, respectively. For those patients with decompensated cirrhosis ( $\mathrm{n}=$ 70 ), the cumulative incidence of HCC was $6.9 \%$ at month 24; four patients developed HCC during the follow-up. The cumulative incidence of mortality or OLT was $12.9 \%$ at month 12 and $17.0 \%$ at month 24 . For 55 patients with decompensated liver function treated with ETV for $\geq 12$ months, improvements from baseline in CTP score and its components (albumin, total bilirubin, prothrombin time) and MELD score were observed $(P<0.05$ for all). CTP class A (score 5 or 6 ) was achieved in $65.5 \%$ (30) of the patients, and improvement in $\mathrm{CTP}(\geq 2$ points reduction) was observed in $49 \%$ (27) of the patients after 12 months of treatment.

\begin{tabular}{llll}
\hline Table 8. One-year Results of Virologic and Biochemical Indices & & & \\
\hline One-year Results & Compensated $(\mathbf{n}=\mathbf{1 4 4})$ & Decompensated $(\mathbf{n}=\mathbf{5 5})$ & P value \\
\hline Change in HBV DNA, (log10 copies/mL) & $6.74 \pm 1.88$ & $6.82 \pm 1.32$ & 0.793 \\
HBV DNA undetectable, No. (\%), (<300 copies/mL by PCR) & $113 / 144(78.5)$ & $49 / 55(89.1)$ & 0.104 \\
HBeAg seroconversion, No. (\%) & $22 / 90(24.4)$ & $6 / 27(22.2)$ & 0.812 \\
HBeAg loss, No. (\%) & $37 / 90(41.1)$ & $13 / 27(48.1)$ & 0.517 \\
ALT normalization, No. (\%) & $108 / 144(75.0)$ & $42 / 55(76.4)$ & 0.535 \\
\hline
\end{tabular}




\subsubsection{Comments}

This cohort study supports the use of ETV as a firstline treatment option for NUC-naive patients with decompensated HBV cirrhosis. Further follow-up of similar studies are needed to identify the optimal treatment for these patients and those with LAM-resistant HBV cirrhosis.

\section{Acknowledgements}

None declared.

\section{Financial Disclosures}

None declared.

\section{Funding/Support}

None declared.

\section{References}

1. European Association For The Study Of The Liver. EASL Clinical Practice Guidelines: management of chronic hepatitis B. J Hepatol. 2009;50(2):227-42.

2. LokASF,McMahon BJ.Chronichepatitis B.Hepatology.2007;45(2):50739.

3. Chang TT, Gish RG, de Man R, Gadano A, Sollano J, Chao YC, et al. A comparison of entecavir and lamivudine for HBeAg-positive chronic hepatitis B. N Engl J Med. 2006;354(10):1001-10.

4. Lai CL, Shouval D, Lok AS, Chang TT, Cheinquer H, Goodman Z, et al. Entecavir versus lamivudine for patients with HBeAg-negative chronic hepatitis B. N Engl J Med. 2006;354(10):1011-20.

5. Marcellin P, Chang TT, Lim SG, Tong MJ, Sievert W, Shiffman ML, et al. Adefovir dipivoxil for the treatment of hepatitis B e antigen-positive chronic hepatitis B. N Engl J Med. 2003;348(9):808-16.

6. Hadziyannis SJ, Tassopoulos NC, Heathcote EJ, Chang TT, Kitis G, Rizzetto M, et al. Adefovir dipivoxil for the treatment of hepatitis B e antigen-negative chronic hepatitis B. $N$ Engl J Med. 2003;348(9):800-7.

7. Lai CL, Gane E, Liaw YF, Hsu CW, Thongsawat S, Wang Y, et al. Telbivudine versus lamivudine in patients with chronic hepatitis B. N Engl J Med. 2007;357(25):2576-88.

8. Marcellin P, Heathcote EJ, Buti M, Gane E, de Man RA, Krastev Z, et al. Tenofovir disoproxil fumarate versus adefovir dipivoxil for chronic hepatitis B. N Engl J Med. 2008;359(23):2442-55.

9. Gish RG, Lok AS, Chang TT, de Man RA, Gadano A, Sollano J, et al. Entecavir therapy for up to 96 weeks in patients with HBeAg-positive chronic hepatitis B. Gastroenterology. 2007;133(5):1437-44

10. Colonno RJ, Rose RE, Pokornowski K, Baldick CJ, Eggers B, Yu D. Four year assessment of ETV resistance in nucleoside-naive and lamivudine refractory patients. J Hepatol. 2007;46(Suppl 1):S294.

11. Yokosuka O, Takaguchi K, Fujioka S, Shindo M, Chayama K, Kobashi $\mathrm{H}$, et al. Long-term use of entecavir in nucleoside-naive Japanese patients with chronic hepatitis B infection. J Hepatol. 2010;52(6):791-9.

12. Liaw YF, Gane E, Leung N, Zeuzem S, Wang Y, Lai CL, et al. 2-Year GLOBE trial results: telbivudine Is superior to lamivudine in patients with chronic hepatitis B. Gastroenterology. 2009;136(2):486-95.

13. Heathcote J, George J, Gordon S, Bronowicki JP, Sperl J, Williams R, et al. Tenofovir disoproxil fumarate (TDF) for the treatment of HBeAgpositive chronic hepatitis B: week $72 \mathrm{tdf}$ data and week 24 adefovir dipivoxil switch data (study 103). Journal of hepatology. 2008;48:S32.

14. Lai CL, Dienstag J, Schiff E, Leung NW, Atkins M, Hunt C, et al. Prevalence and clinical correlates of YMDD variants during lamivu- dine therapy for patients with chronic hepatitis B. Clin Infect Dis. 2003;36(6):687-96.

15. Snow-Lampart A, Chappell BJ, Curtis M, Zhu Y, Heathcote EJ, Marcellin P. Week 96 resistance surveillance for HBeAg positive and negative subjects with chronic HBV infection randomized to receive tenofovir DF 300 mg qd. Hepatology. 2008;48(Suppl 1):745A.

16. Hadziyannis SI, Tassopoulos NC, Heathcote EJ, Chang TT, Kitis G, Rizzetto $\mathrm{M}$, et al. Long-term therapy with adefovir dipivoxil for HBeAg-negative chronic hepatitis B. N Engl J Med. 2005;352(26):267381.

17. Perrillo R, Hann HW, Mutimer D, Willems B, Leung N, Lee WM, et al. Adefovir dipivoxil added to ongoing lamivudine in chronic hepatitis B with YMDD mutant hepatitis B virus. Gastroenterology. 2004;126(1):81-90

18. Peters MG, Hann Hw H, Martin P, Heathcote EJ, Buggisch P, Rubin R, et al. Adefovir dipivoxil alone or in combination with lamivudine in patients with lamivudine-resistant chronic hepatitis B. Gastroenterology. 2004;126(1):91-101.

19. Schiff ER, Lai CL, Hadziyannis S, Neuhaus P, Terrault N, Colombo $\mathrm{M}$, et al. Adefovir dipivoxil therapy for lamivudine-resistant hepatitis B in pre- and post-liver transplantation patients. Hepatology. 2003;38(6):1419-27.

20. Heathcote J, George J, Gordon S, Bronowicki JP, Sperl J, Williams R, et al. 72 Tenofovir Disoproxil Fumarate (Tdf) For The Treatment Of Hbeag-Positive Chronic Hepatitis B: Week 72 Tdf Data And Week 24 Adefovir Dipivoxil Switch Data (Study 103). Journal of hepatology. 2008;48:S32.

21. Marcellin P, Jacobson I, Habersetzer F, Senturk H, Andreone P, Moyes C, et al. 57 Tenofovir Disoproxil Fumarate (Tdf) For The Treatment Of Hbeag-Negative Chronic Hepatitis B: Week 72 Tdf Data And Week 24 Adefovir Dipivoxil Switch Data (Study 102).J Hepatol. 2008;48:S26.

22. Zoulim F, Perrillo R. Hepatitis B: reflections on the current approach to antiviral therapy.J Hepatol. 2008;48(Suppl 1):S2-19.

23. Lampertico P, Vigano M, Manenti E, Iavarone M, Colombo M. Add-on adefovir prevents the emergence of adefovir resistance in lamivudine-resistant patients: A 4-year study. J Hepatol. 2008;48:S259.

24. Cooksley WG, Piratvisuth T, Lee SD, Mahachai V, Chao YC, Tanwandee T, et al. Peginterferon alpha-2a (40 kDa): an advance in the treatment of hepatitis B e antigen-positive chronic hepatitis B. J Viral Hepat. 2003;10(4):298-305.

25. Janssen HL, van Zonneveld M, Senturk H, Zeuzem S, Akarca US, Cakaloglu Y, et al. Pegylated interferon alfa-2b alone or in combination with lamivudine for HBeAg-positive chronic hepatitis B: a randomised trial. Lancet. 2005;365(9454):123-9.

26. Lau GK, Piratvisuth T, Luo KX, Marcellin P, Thongsawat S, Cooksley $\mathrm{G}$, et al. Peginterferon Alfa-2a, lamivudine, and the combination for HBeAg-positive chronic hepatitis B. N Engl J Med. 2005;352(26):268295.

27. Marcellin P, Lau GK, Bonino F, Farci P, Hadziyannis S, Jin R, et al. Peginterferon alfa-2a alone, lamivudine alone, and the two in combination in patients with HBeAg-negative chronic hepatitis B. NEngl JMed. 2004;351(12):1206-17.

28. Levin J, editor. Efficacy and Safety of Entecavir Versus Adefovir in Chronic Hepatitis B Patients with Evidence of Hepatic Decompensation 60th Annual Meeting of the American Association for the Study of Liver Diseases; 2009 October 30-November 3; Boston. USA; Hynes Convention Center.

29. Liaw YF, Lee CM, Akaraca U, Papatheodoridis G, Wong F, Chang TT, et al. A Phase 2, Double-Blind, Randomized Study Comparing The Safety of Tenofovir Disoproxil Fumarate (TDF), Emtricitabine Plus TDF (Truvada, TVD) and Entecavir (ETV) in Subjects with Decompensated Chronic Hepatitis B Liver Disease. 60th Annual Meeting of the American Association for the Study of Liver Diseases; 2009 October 30-November 3; Boston, MA, Hynes Convention Center. 2009. p. Abstract 222.

30. Shim JH, Lee HC, Kim KM, Lim YS, Chung YH, Lee YS, et al. Efficacy of entecavir in treatment-naive patients with hepatitis B virus-related decompensated cirrhosis. J Hepatol. 2010;52(2):176-82. 